\title{
Detection of viable Plasmodium ookinetes in the midguts of Anopheles coluzzi using PMA-qrtPCR
}

\author{
Tibebu Habtewold ${ }^{1,2^{*}}$, Zoe Groom ${ }^{1,3}$, Luc Duchateau ${ }^{2}$ and George K. Christophides ${ }^{1}$
}

\begin{abstract}
Background: Mosquito infection with malaria parasites depends on complex interactions between the mosquito immune response, the parasite developmental program and the midgut microbiota. Simultaneous monitoring of the parasite and bacterial dynamics is important when studying these interactions. PCR based methods of genomic DNA (gDNA) have been widely used, but their inability to discriminate between live and dead cells compromises their application. The alternative method of quantification of mRNA mainly reports on cell activity rather than density.

Method: Quantitative real-time (qrt) PCR in combination with Propidium Monoazide (PMA) treatment (PMA-qrtPCR) has been previously used for selectively enumerating viable microbial cells. PMA penetrates damaged cell membranes and intercalates in the DNA inhibiting its PCR amplification. Here, we tested the potential of PMA-qrtPCR to discriminate between and quantify live and dead Plasmodium berghei malarial parasites and commensal bacteria in the midgut of Anopheles coluzzii Coetzee \& Wilkerson 2013 (formerly An. gambiae M-form).

Results: By combining microscopic observations with reverse transcriptase PCR (RT-PCR) we reveal that, in addition to gDNA, mRNA from dead parasites also persists inside the mosquito midgut, therefore its quantification cannot accurately reflect live-only parasites at the time of monitoring. In contrast, pre-treating the samples with PMA selectively inhibited qrtPCR amplification of parasite gDNA, with about 15 cycles (Ct-value) difference between PMA-treated and control samples. The limit of detection corresponds to 10 Plasmodium ookinetes. Finally, we show that the PMA-qrtPCR method can be used to quantify bacteria that are present in the mosquito midgut.

Conclusion: The PMA-qrtPCR is a suitable method for quantification of viable parasites and bacteria in the midgut of Anopheles mosquitoes. The method will be valuable when studying the molecular interactions between the mosquito, the malaria parasite and midgut microbiota.
\end{abstract}

Keywords: Anopheles coluzzii, Propidium monoazide, Plasmodium, Midgut invasion, Commensal microbiota

\section{Background}

The first hours after ingestion of an infectious blood meal by the mosquito vector are key for sucssesful infection with malaria parasites. This period of the Plasmodium lifecycle involves gametogenesis, fusion of the male and female gametes, zygote development and differentiation to motile ookinete, ookinete traversal of the peritrophic membrane and the midgut epithelium and transformation to oocyst. Importantly, this period is marked by a major

\footnotetext{
* Correspondence: t.habtewold@ic.ac.uk

'Department of Life Sciences, Imperial College London, London, UK 2Department of Comparative Physiology and Biometrics, University of Ghent, Ghent, Belgium

Full list of author information is available at the end of the article
}

drop of parasite numbers. The rate of parasite losses during the ookinete to oocyst transition in An. gambiae is shown to reach 50, 41 and 69 folds for $P$. berghei [1], P. yoelii $[2,3]$ and P. falciparum [4], respectively.

Inside the mosquito blood bolus, Plasmodium parasites are found in close proximity with the mosquito midgut microbiota. The Anopheles mosquito midguts harbour numerous genera of microbiota, including Pseudomonas, Aeromonas, Asaia, Comamonas, Elizabethkingia, Enterobacter, Klebsiella, Pantoea, Serratia and others [5]. These microbiota proliferate dramatically and reach a peak at approximately $24-30 \mathrm{~h}$ post blood meal, before falling back to pre-blood meal levels [6]. The microbiota in the mosquito midgut play a significant role in malaria 
transmission dynamics. It has been shown that Plasmodium infection intensity is increased when mosquitoes are treated with antibiotics and this phenotype is reversed when microbiota are reconstituted (e.g. [7, 8]). Interaction of the microbiota with Plasmodium development appears to be exclusive to certain strains of gram-negative bacteria [9]. Currently, the mechanisms underlying the apparent bacterial inhibition of Plasmodium development are not fully elucidated; they are thought to involve parasite killing both directly and through induction of mosquito immune responses [9-12]. Induction of immune responses either through the gut microbiota or through direct parasite recognition plays a major role in vector competence.

Microscopic examination of mosquito midgut epithelium remains the gold standard for determining Plasmodium infection intensity. Dissection and microscopic examination of mosquito midguts is typically performed 5-7 days after infection, in order to allow the blood meal bolus to clear and the oocyst to increase in size becoming detectable. However, by this time, the gut bacterial population and mosquito innate immune activity have subsided to a normal state. Thus, microscopy does not allow the simultaneous assessment of the parasite, bacterial and immune response dynamics. Quantitative real-time PCR (qrtPCR) of genomic DNA (gDNA) are currently used as a fast, sensitive, and specific molecular tool for the detection and quantification of pathogens, including Plasmodium parasites in the mosquito $[13,14]$. However, false-positive results due to amplification of gDNA from dead cells are a major drawback. In environmental microbiology, an alternative method involves mRNA-based qrtPCR for selective detection of viable cells $[15,16]$. This technique takes advantage of the rapid mRNA degradation compared to gDNA. However, mRNA does not accurately represent bacterial cell density but mostly refers to bacterial cell growth and proliferation.

A diagnostic technique that precludes detection of DNA from dead cells involves pre-treatment of samples with propidium monoazide (PMA) and subsequent PCR analysis of gDNA [17]. This method is referred as viability PCR (v-PCR). PMA has high affinity to double stranded DNA and, upon photolysis using bright white light, reacts strongly with hydrocarbons of the bound DNA inducing permanent modification and rendering it inaccessible to DNA polymerase [18]. The technique exploits the fact that PMA not only binds to free floating DNA but also the DNA of dead cells as it penetrates the damaged or permeable membranes of dead cells but not the intact membranes of viable cells. The PMA-qrtPCR technique has been increasingly used for the selective detection of viable bacteria $[19,20]$, fungi [21] and protozoa [22]. Here, we evaluated the potential of the PMA-qrtPCR technique to simultaneously quantify viable Plasmodium parasites and bacterial microbiota in the mosquito midgut. This method could be used to investigate the interactions between the mosquito vector, the gut microbiota and the Plasmodium parasite during the first critical hours of mosquito infection.

\section{Methods}

\section{Ethics statement}

The protocol for infecting mice with $P$. berghei and P. yoelii was approved and carried out at the Imperial College London under the UK Home Office License PPL70/7185 awarded in January 2010.

\section{Mosquito colonies and maintenance}

The Anopheles gambiae strain N'gousso M-form (a laboratory-strain colonized in 2006 from field mosquitoes collected around Yaoundé, Cameroon), now formally named as Anopheles coluzzii Coetzee \& Wilkerson 2013 [23], were used in these experiments. The mosquitoes were reared and maintained at $27{ }^{\circ} \mathrm{C}, 70 \%$ relative humidity, subject to a $12 \mathrm{~h}$ light/dark cycle. Adult mosquitoes were fed on $10 \%$ sucrose cotton pads.

\section{Plasmodium strains and maintenance}

Green Florescent Protein (GFP)-Plasmodium berghei (ANKA 2.34 strain) [24] or GFP-Plasmodium yoelii (17X strain) [25] parasite lines were used throughout this study. The parasite lines were maintained by serial passage in 8-12 week-old female TO mice (Harlan, UK).

\section{Mosquito infection with plasmodium}

Female mosquitoes, 3-5 days old, were infected with Plasmodium parasites using two methods: (1) direct feeding on gametocytemic mice as previously described [26] or (2) using the membrane feeder system with ookinetes. For the ookinete culture, mice were injected with $P$. berghei or P. yoelii infected 3 days after phenyl hydrazine treatment to encourage reticulocyte formation. 3 days post infection, $1 \mathrm{ml}$ of blood was drawn from the mice and immediately transferred to a vented tissue culture flask containing $30 \mathrm{~mL} 80 \%(\mathrm{v} / \mathrm{v})$ complete ookinete culture medium (RPMI1640 (Sigma)). The culture was incubated at $21{ }^{\circ} \mathrm{C}$ in air for $24 \mathrm{~h}$ ( $48 \mathrm{~h}$ for $P$. yoelii). The number of mature ookinetes was determined with a haemocytometer. Blood meal serum, containing approximately 800 ookinetes $/ \mu$ l, was offered to mosquitoes for feeding.

\section{Injection of mosquitoes with ookinetes}

Mosquitoes were directly injected with viable or heat inactivated ookinetes (heated at $42{ }^{\circ} \mathrm{C}$ for $15 \mathrm{~min}$ ) into their hemolymph. Each mosquito received 400 ookinetes in freshly prepared ookinete culture media. Mosquitoes challenged with $P$. berghei and $P$. yoelii parasites 
in all the above methods were maintained at $21^{\circ} \mathrm{C}$ and $24{ }^{\circ} \mathrm{C}$, respectively.

\section{Dissecting mosquito midgut and harvesting gut content} Midgut contents of infected mosquitoes were dissected on ice cold PBS + BSA (2\%). For harvesting the gut contents, a midgut was placed in $20 \mu \mathrm{l}$ of PBS and a longitudinal incision was made on the gut to draw out its contents into the PBS. The resulting sheet of midgut tissue and the gut contents were used in subsequent microscopic examination or nucleic acid extraction.

\section{Preparation of samples for microscopic examination}

Midgut sheets were immunostained with Pbs28 antibody conjugated with 13.1-Cy3 dye for $30 \mathrm{~min}$ on ice and then examined under florescent microscopy to detect or enumerate dead and viable ookinetes. Dead ookinetes were negative for GFP. Alternatively, midguts were subjected to dual staining using propidium monoazide (PMA) and Syber Green (Sb) I dyes. Cells with compromised membranes (considered as inactive) were positive for red florescence arising from PMA, whereas the viable cells remained green.

\section{Total RNA (tRNA) extraction}

Total RNA was extracted from whole mosquito or midgut content. To extract tRNA from a whole mosquito, 10 mosquitoes were transferred into $2 \mathrm{ml}$ Safe-Lock RNA/DNA extraction RNase free tube containing $90 \mu \mathrm{l}$ lysis buffer ( $3 \mathrm{mg} / \mathrm{ml}$ lysozyme in TE) and $\sim 25 \mathrm{ng}$ acid washed glass beads. The mixture was then incubated in a thermostatic mixer for $10 \mathrm{~min}$ at $37{ }^{\circ} \mathrm{C}$ at $300 \mathrm{rpm}$, and for an additional $10 \mathrm{~min}$ after adding $10 \mu \mathrm{l}$ proteinase K. Buffer RLT $(350 \mu \mathrm{l})$ was added to the mixture, and then homogenised using Precellys ${ }^{\circ} 24$ tissue homogeniser. The homogenate was spiked with 106 copies of control RNA template transcribed from the plasmid pAW109 (GeneAmp RNA kit) as an internal standard. Total RNA was also extracted from the midgut content of mosquito. In this case, the midgut was emptied into $20 \mu \mathrm{l} \mathrm{PBS}$. The gut content together with PBS was transferred to an RNase free tube containing the lysis buffer $(80 \mu \mathrm{l})$. After incubation (see above), the RLT buffer $(350 \mu \mathrm{l})$ was added to the mixture and the mixture was spiked with control RNA template. For all types of samples in RLT buffer, QIAGEN RNeasy ${ }^{\circ}$ Mini kit was used to extract tRNA following the kit protocol.

\section{Sample preparation for treatment with PMA}

In vitro ookinete culture, bacterial culture from mosquito gut content, midgut epithelial tissue of mosquitoes, gut content and whole mosquitoes samples were prepared for PMA treatment as follows. The total volume of in vitro ookinete culture, containing a known number of mature ookinetes, was adjusted to $500 \mu \mathrm{l}$ to prepare for PMA treatment. Bacterial culture was prepared for the PMA treatment as follows. The midgut bolus was removed from mosquitoes that had obtained a naive bloodmeal $24 \mathrm{~h}$ before the dissection and was directly inoculated into LB medium $(5 \mathrm{ml})$ and incubated over night at $37{ }^{\circ} \mathrm{C}$. A $500 \mu \mathrm{l}$ aliquot of the bacterial culture was removed and heated at $100{ }^{\circ} \mathrm{C}$ for $10 \mathrm{~min}$ before PMA treatment. The control sample was left unheated. Ookinetes embedded in the midgut epithelia tissues were prepared for PMA treatment. First, midguts were dissected $(n=10)$ on ice cold PBS + PMA and were transferred into $500 \mu \mathrm{l}$ ice cold PBS-BSA (2 \%) after removing blood boluses. The bloodmeal bolus in PBS + BSA was transferred into a separate tube for PMA treatment. To prepare whole mosquitoes for PMA treatment, first a longitudinal incision was made in the abdomen to expose the interior including the midgut that was dissected onto ice cold PBS + BSA. Then an additional longitudinal incision was made into the midgut. All the mosquito tissue, including the blood meal bolus, was transferred to a tube and the volume was adjusted to $500 \mu \mathrm{l}$ with PBS-BSA. Finally, the sample was placed on a rocker for $1 \mathrm{~h}$ in the cold and the circular sheath was carefully removed with fine forceps.

\section{PMA treatment and genomic DNA (gDNA) extraction}

Procedures for PMA treatment described previously $[19,27]$ were used with minor modification. Briefly, PMA stock solution $(20 \mathrm{mM}$ in $20 \%$ dimethyl sulfoxide; Biotium Inc., Hayward, CA) was added at a concentration of $200 \mathrm{mM}$. The sample-PMA mixture was incubated in the dark for 20 min. To cross link PMA to DNA molecules, the samples were placed horizontally on ice on a shaker and exposed for $5 \mathrm{~min}$ to a $650 \mathrm{~W}$ halogen lamp at a distance of $20 \mathrm{~cm}$. Genomic DNA extraction was performed on the PMA treated samples using a QIAGEN DNeasy ${ }^{\circ}$ Blood \& Tissue kit. After PMA treatment, the sample was spun at $3000 \mathrm{rpm}$ for $10 \mathrm{~min}$ at a cold temperature, and the supernatant was replaced by $180 \mu \mathrm{l}$ of lysis buffer from the kit. This mixture was incubated for $30 \mathrm{~min}$ at room temperature and then for $30 \mathrm{~min}$ at $55^{\circ} \mathrm{C}$ after adding $20 \mu \mathrm{l}$ proteinase $\mathrm{K}$. The mixture was then homogenised using Precellys ${ }^{\circ} 24$ tissue homogeniser. Before proceeding to the gDNA extraction, the homogenate was spiked with 106 copies of control cDNA synthesised from RNA template transcribed from the plasmid pAW109 (GeneAmp ${ }^{\circ}$ RNA kit) as an internal standard. The QIAGEN DNeasy Blood \& Tissue kit protocol was followed to extract gRNA.

Reverse transcription polymerase chain reaction (RT-PCR) Expression of Secreted Ookinete Adhesive Protein (SOAP) gene by Plasmodium ookinetes was assessed using RNA isolated from the whole mosquito or from the blood meal 
bolus at specified time points. RT-PCR was performed using the QIAGEN One-step RT-PCR method which ensures a high specificity and sensitivity and minimises sample loss and contamination. Briefly, tRNA samples $(25 \mu \mathrm{l})$ were added to an RT-PCR master mix containing 5x OneStep RT-PCR Enzyme Buffer $10 \mu \mathrm{l}$, dNTP mix $(10 \mathrm{mM}) 2 \mu \mathrm{l}, 5 \mathrm{x}$ Q-Solution $10 \mu \mathrm{l}$, gene specific primers $(10 \mu \mathrm{M}) 0.5 \mu \mathrm{l}$ each and One-step RT-PCR Enzyme mix $2 \mu \mathrm{l}$ to make a final volume of $50 \mu \mathrm{l}$. The primers and their sequences used for amplification of the SOAP gene in both the parasite species were Forward, TCGAAGGAG CAAGGAAAAATTCC and Reverse, ATGAACAGCTA CATTCTTCGGTC. The amplified product was a 438 bp fragment. Exogenous pAW109 RNA (106), co-extracted with the sample RNAs, served as an invariant control. The GeneAmp ${ }^{\circ}$ RNA kit primers for pAW109 control RNA were used to amplify a product of $308 \mathrm{bp}$, primer DM151, GTCTCTGAATCAGAAATCCTTCTATC and Primer DM152, ATGTCAAATTTCACTGCTTCATCC were also used. The reaction mixture was first incubated at $50{ }^{\circ} \mathrm{C}$ for $30 \mathrm{~min}$ to reverse transcribe tRNA, followed by heating at $95{ }^{\circ} \mathrm{C}$ for 15 min to activate DNA Polymerase, deactivate Omniscript and Reverse Transcriptase, and denature the cDNA template. Initial heating was followed by 30 cycles of $94{ }^{\circ} \mathrm{C}$ for $30 \mathrm{~s}, 56^{\circ} \mathrm{C}$ for $30 \mathrm{~s}$ and $72{ }^{\circ} \mathrm{C}$ for $30 \mathrm{~s}$. The final elongation stage was carried out at $72{ }^{\circ} \mathrm{C}$ for $4 \mathrm{~min}$. RT-PCR products were analysed by agarose $1 \%$ gel electrophoresis.

\section{Real-time PCR (QRT-PCR)}

A series of qrtPCR assays were performed on the gDNA that was extracted from In vitro ookinete culture, bacterial culture from the mosquito gut content, the midgut epithelial tissue of mosquitoes, the gut content and the whole mosquito to determine the relative abundance of Plasmodium genes or gut bacterial genes. Set primer pairs used to amplify the SOAP, CTRP or GFP gene were SOAP Forward CCAAAACAACAGGCCAAGAG and SOAP Reverse AACATCGGCCAATGGATTAC, CTRP Forward TGCAATGATGTTTGTGGTGATTT and CTRP Reverse TGGTGATACATTTCTGGTTCTTATT CTT, GFP Forward CCTGTCCTTTTACCAGACAACCA and GFP Reverse GGTCTCTCTTTTCGTTGGGATCT. Universal 16S bacterial primers sets (357f CTCCTACGG GAGGCAGCAG and 519r GTTTACCGCGGCAGCTG) were used to amplify a $162 \mathrm{bp}$ fragment. The PCR reactions were performed in a total volume of $20 \mu \mathrm{l}$, containing $2 \mu \mathrm{l}$ gDNA, $10 \mu \mathrm{l}$ of $2 \mathrm{x}$ SYBR $^{\circ}$ premix Ex Taq (Takara), $0.2 \mu \mathrm{M}$ of each forward and reverse primer specific to target genes or internal standard and 0.4 $\mu$ l Rox reference dye (50x). Amplification and detection of the florescence signal was carried out using an Applied Biosystems 7500 Fast RealTime PCR system. The PCR cycling program consisted of an initial denaturation at $95{ }^{\circ} \mathrm{C}$ for $20 \mathrm{~s}$, followed by
40 cycles of $95{ }^{\circ} \mathrm{C}$ for $3 \mathrm{~s}$ and $60{ }^{\circ} \mathrm{C}$ for $30 \mathrm{~s}$. Each target was quantified in duplicate and the values were normalized by the data obtained with An. gambiae rRNA gene (AgS7) as internal standards. The primer sequences for the internal standard AgS7 gene were: forward, GTGC GCGAGTTGGAGAAGA and reverse, ATCGGTTTG GGCAGAATGC.

A standard curve was generated after qrtPCR analysis on a serial dilution of ookinetes from in vitro culture to calculate the limit of detection (LoD) and limit of quantification (LoQ) for PMA-qrtPCR techniques as described in a previous study [28].

\section{Results}

Microscopic and RT-PCR detection of ookinetes in the blood bolus

Direct microscopic examination of smears of midgut contents in P. berghei (Fig. 1a) and P. yoelii (Fig. 1b) infected $A$. gambiae mosquitoes confirmed that both zygotes and mature ookinetes were present in the gut lumen at $18 \mathrm{~h}$ post infection (hpi), while mature ookinetes were only observed at $24 \mathrm{hpi}$. All the ookinetes were physically cleared from the gut lumen by $48 \mathrm{hpi}$. However, RT-PCR performed on total RNA extracted from the mosquito midgut content showed that transcripts of the ookinete-specific gene SOAP were detectable until 48 hpi (Fig. 1c). The physical as well as molecular detection of $P$. yoelii ookinetes in the mosquito gut content were considerably more transient compared to $P$. berghei. This is expected as the $P$. yoelii infected mosquitoes were kept at a higher temperature $\left(24{ }^{\circ} \mathrm{C}\right)$ compared to $P$. berghei $\left(21^{\circ} \mathrm{C}\right)$. Mature P. yoelii ookinetes were observed in midgut contents harvested at $18 \mathrm{hpi}$, but only ookinete remains were detected at 24 hpi. As with $P$. berghei, $P$. yoelii SOAP transcripts were still detectable at $24 \mathrm{hpi}$ and only cleared by $48 \mathrm{hpi}$ (Fig. 1d). These data indicate that ookinete-specific transcripts persist in the blood bolus even in the absence of viable ookinetes.

\section{Persistence of ookinete mRNA in the mosquito midgut and hemolymph}

To determine the persistence of ookinete mRNA in mosquito tissues, heat-killed $\left(42{ }^{\circ} \mathrm{C}\right.$ for $\left.30 \mathrm{~min}\right)$ or live in vitro cultured ookinetes were either added to mouse blood and offered to female An. gambiae mosquitoes as a blood meal via membrane feeding or injected directly into the mosquito hemolymph. Approximately 800 ookinetes were delivered in each mosquito with either of the two methods. RT-PCR analysis of SOAP transcripts was performed using total RNA extracted from whole mosquitoes at $0,3,6,12,18,24$ and 48 hpi. The results showed prolonged persistence of SOAP transcripts from both heat-killed and live $P$. berghei ookinetes compared 


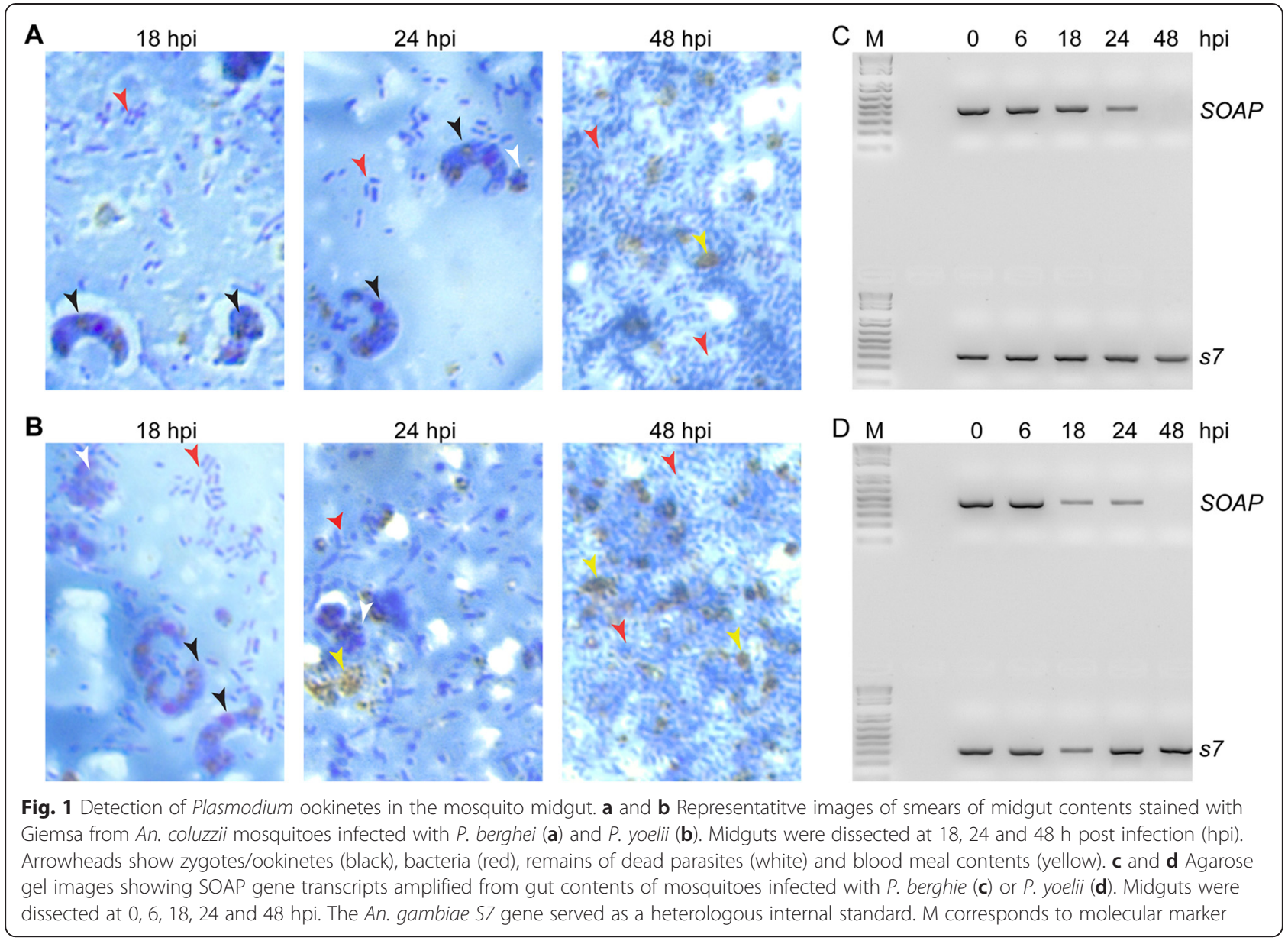

to P. yoelii ookinetes (Fig. 2). This difference is attributable to the difference in temperature between these two infection models and consequently the slower metabolic rate of mosquitoes at $21{ }^{\circ} \mathrm{C}(P$. berghei) compared to $24{ }^{\circ} \mathrm{C}$ (P. yoelii). For heat-killed ookinetes, SOAP transcripts persisted in mosquito tissues up to $24 \mathrm{hpi}$ and 18 hpi for P. berghei and P. yoelii ookinetes, respectively, whereas transcripts from live ookinetes were detectable until 48 hpi. The slow mRNA decay rate suggests that transcript abundances measured by PCR methods are not fully reflective of viable parasites.

\section{PMA-induced inhibition of DNA amplification from dead parasites and bacteria}

Propidium monoazide (PMA) has been increasingly used for the treatment of microbiological samples to exclude PCR signals from non-viable cells, but this has not been tested to date in investigations involving Plasmodium. We confirmed that PMA can stain dead P. berghei ookinetes prepared from an ookinete culture and heat-killed at $42{ }^{\circ} \mathrm{C}$ for $30 \mathrm{~min}$ before incubating them with PMA (Fig. 3a). Next, we stained ookinetes sampled directly from an overnight culture that was thought to include both live and dead cells. The ookinetes were incubated with a mixture of PMA and SYBR green that also stains nucleic acids but, unlike PMA, actively enters live cells. Fluorescent microscopic observations confirmed that some ookinetes are stained only with PMA, while most of the ookinetes are stained only with SYBR green (Fig. 3b). We applied the same protocol to An. gambiae midguts tissues infected $24 \mathrm{~h}$ earlier with $P$. berghei. The results showed that SYBR and PMA could be used to discriminate between live and dead parasites, respectively (Fig. 3c). Bacteria present in the blood meal bolus were also differentially stained with SYBR and PMA (Fig. 3d).

We assessed whether incorporation of PMA in nucleic acids could inhibit the PCR amplification rates of mRNA transcripts that derived from dead P. berghei parasites. Genomic DNA (gDNA) was prepared from heat-killed parasites from an overnight in vitro ookinete culture treated with PMA or control solution, and the abundances of SOAP, CTRP and GFP (the parasite line used carried a GFP expression cassette) amplicons were determined by quantitative real-time PCR (qrtPCR). The parasite gDNA samples were spiked with equal amounts 

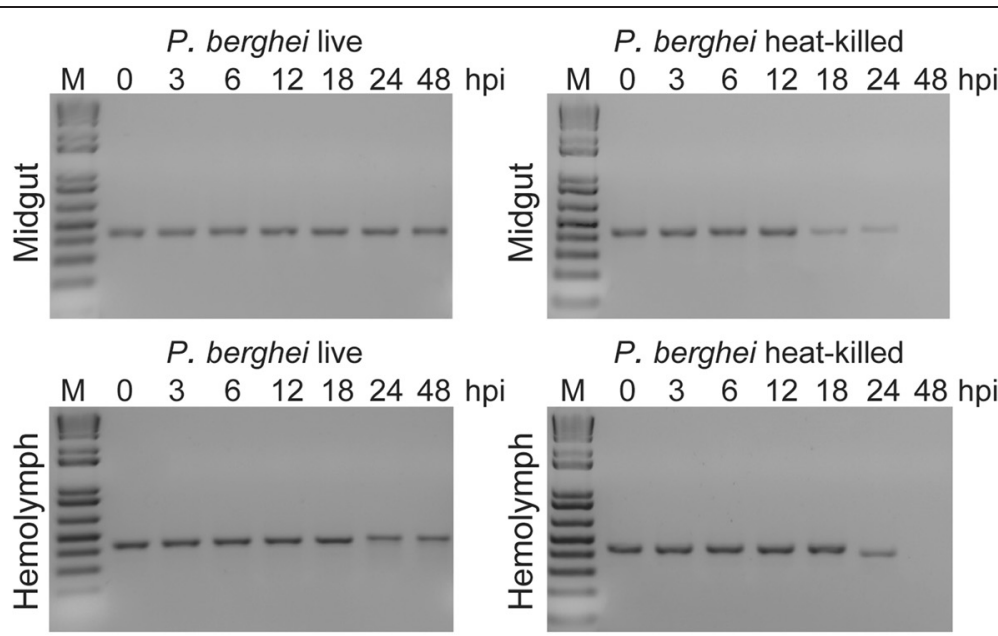

$P$. berghei heat-killed

P. yoelii live
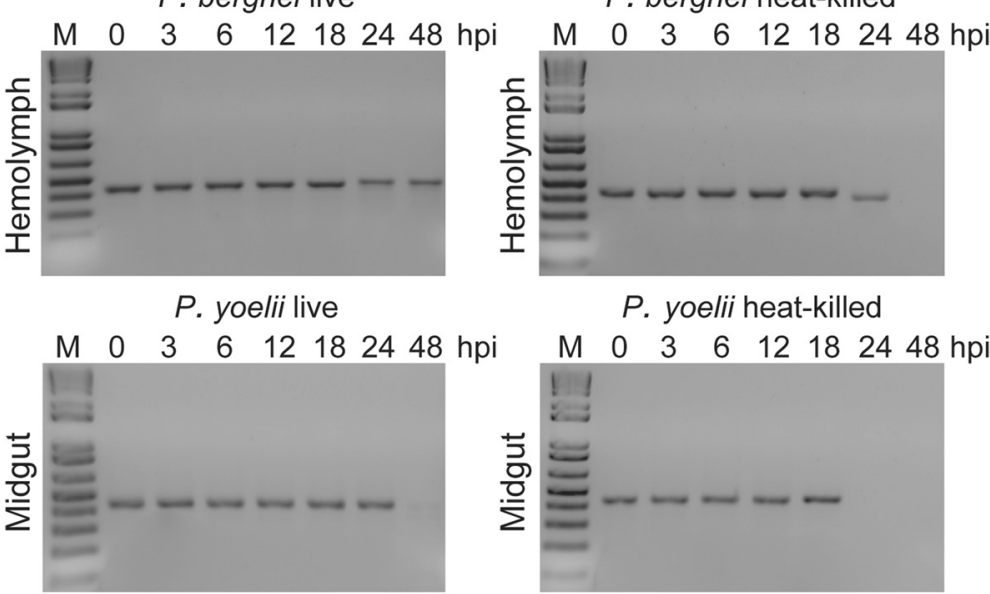

$P$. yoelii heat-killed

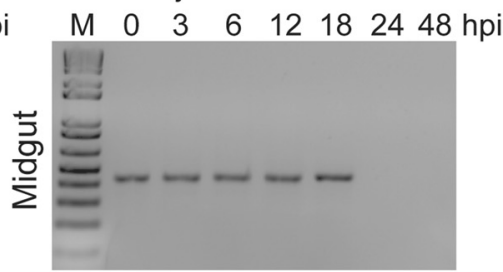

P. yoelii live

$P$. yoelii heat-killed
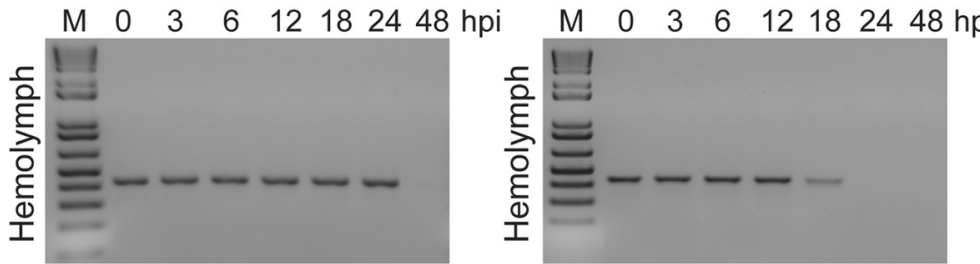

Fig. 2 SOAP mRNA persistence in the An. coluzzii midgut and hemolymph. Agarose gel electrophoresis images of Plasmodium SOAP gene transcrips in An. coluzzii mosquitoes. Heat-killed or live P. berghei or $P$. yoelii ookinetes were delivered to mosquitoes either by addition to the blood meal or injection into the haemolymph. RT-PCR assays were carried our on whole mosquito RNAs prepared at $0,3,6,12,18,24$ and $48 \mathrm{~h}$ post ookinete ingestion or injection (hpi)

of An. gambiae gDNA and amplification of the $\mathrm{S} 7$ gene served as a heterologous internal standard. The results showed that PMA treatment significantly inhibited the qrtPCR detection of SOAP, CTRP or GFP genes in dead ookinetes (Fig. 4a). We observed a similar inhibitory effect of PMA on qrtPCR assays for bacterial 16S rRNA gene in the gDNA from total culturable bacteria in the mosquito midgut content (Fig. 4b).

Next, we tested the PMA inhibitory effect on parasite detection in mosquitoes by comparing the level of

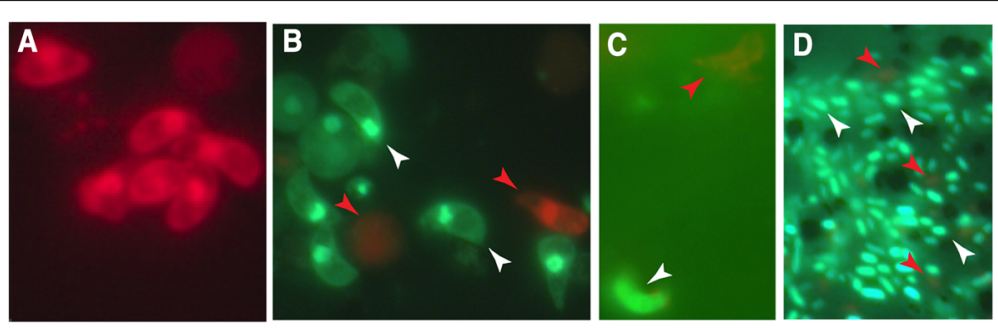

Fig. 3 Differential staining of live and dead ookinetes and bacteria in the mosquito midgut. a Heat killed ookinetes an overnight ookinete culture stained with a mixture of PMA and SYBR solution. $\mathbf{b}$ Live and dead parasites from an overnight ookinete culture stained with a mixture of PMA and SYBR solution. $\mathbf{c}$ Live and dead P. berghei ookinetes in the mosquito midgut epithelium at 48 hpi. $\mathbf{d}$ Live and dead bacteria present in midgut contents. Live cells are stained with SYBR green (white arrowheads), while dead cells are stained with PMA (red arrowheads) 

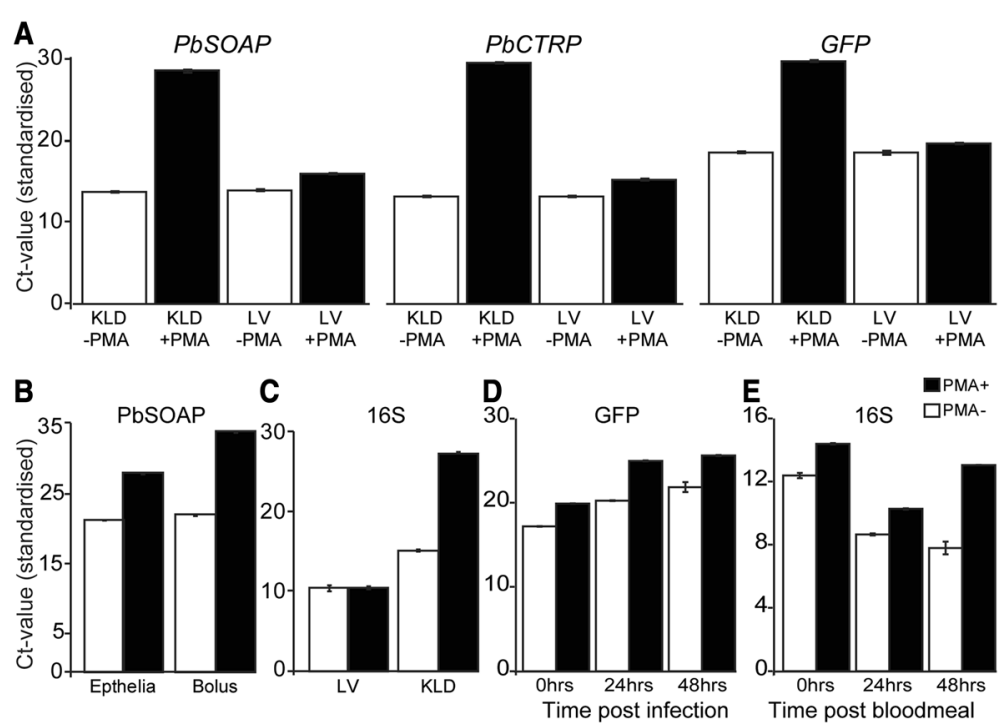

Fig. 4 PMA-qrtPCR investigation of the inhibition of DNA amplification in killed ookinetes or bacteria during PCR. a Average cycle threshold (ct-value) for PbSOAP PbCTRP and PbGFP CON genes in heat inactivated (KLD) or live (LV) ookinetes from overnight gametocyte culture that were pre-treated with PMA. Inactivated and live ookinetes without PMA were used as a control. Samples, before DNA extraction, were spiked with an equal volume of Anopheles coluzzii homogenate with the aim to use the mosquito 57 gene as a heterologous internal standard. b Average ct-values for the PbGFP gene in the midgut contents or gut epithelium tissue from mossquitoes $24 \mathrm{~h}$ pi with $P$. berghei parasite. c Average ct-values for bacterial $16 \mathrm{~S}$ rRNA gene from heat inactivated samples and live bacteria cultured from mosquito midgut contents (24 h after bloodmeal). Samples without PMA treatment served as a control. $\mathbf{d}$ Ct-values for PbGFP genes from gDNA samples that were extracted from mosquitoes that obtaned $P$. berghei ookinetes in their bloodmeal. e Ct-values for $16 \mathrm{~S}$ rRNA genes in gDNA extracted from mosquitoes that obstained naïve bloodmeal. An. gambiae $S 7$ gene served as a heterologous internal standard for all the PMA-qrtPCR reactions that were performed using samples that were isolated from mosquito tissues. Error bars represent standard deviation from at least two independent assays. Each qrPCR experiment repeated three times

reduction of qrtPCR signal (increase in Ct-value) in the midgut contents and midgut epithelial tissues of mosquitoes that had been infected 24 h earlier with $P$. berghei parasites. The qrtPCR analysis of the PbSOAP gene showed an increased Ct-value in gut content or gut epithelial tissue samples that were treated with PMA when compared to controls (Fig. 4c). The Ct-value was higher for PMA treated samples at $24 \mathrm{~h}$ pi compared to $0 \mathrm{~h}$ pi, but there was no further increase from $24 \mathrm{~h}$ to $48 \mathrm{~h}$, suggesting the PMA treatment inhibited DNA amplification from dead ookinetes and also from those ookinetes that were eventually killed.

\section{Detection and quantification of ookinetes using PMA qrt-PCR}

The efficiency of PMA-qrtPCR for detecting and quantifying Plasmodium ookinetes was tested using 10-fold serial dilutions of $P$. berghei ookinetes cultured in vitro, from $2.5 \times 10^{6}$ to $2.5 \times 10^{-1}$. Samples were spiked with equal volumes of mosquito tissue homogenates before proceeding to gDNA extraction. In two independent PMA-qrtPCR experiments, the resulting $\mathrm{Ct}$-values were standardized to the An. gambiae $\mathrm{S} 7$ gene in the corresponding experiment. Averaged $\mathrm{Ct}$-values corresponding to each dilution were plotted against the ookinete numbers in the respective dilution. Standard curves were linear for the serial dilution range (Fig. 5). The limit of quantification (LoD) for the PMA-qrtPCR was $\sim 25$ ookinetes.

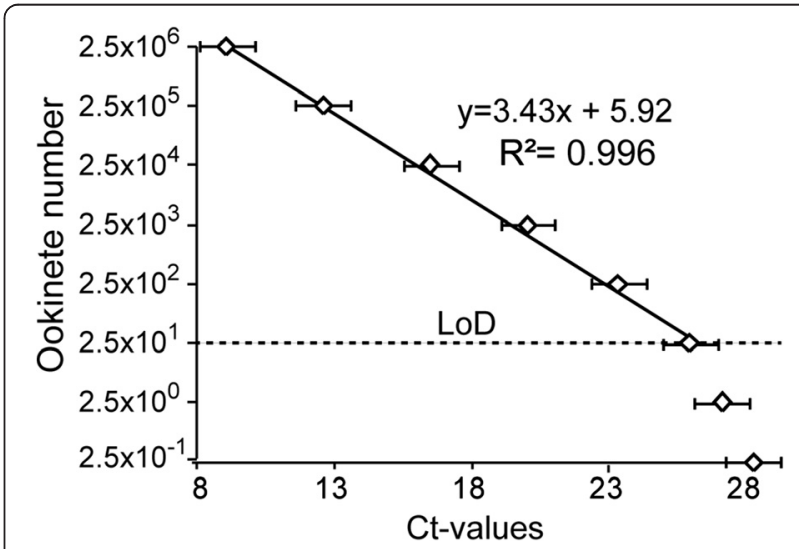

Fig. 5 Ookinete detection efficiency using PMA-qrtPCR. Standard curve for quantifying $P$. berghei ookinetes using 10-fold serially diluted parasite cultures. Ookinete numbers are plotted against cycle threshold (Ct) values. Duplicate samples from two independent experiments were quantified. The dotted line indicates the limit of detection (LoD) 


\section{Discussion and Conclusions}

In this study, we performed a series of experiments aimed to establish an efficient PCR based method to detect and quantify the success of Plasmodium parasite infection of the mosquito midgut. The majority of Plasmodium ookinetes are killed during the first $32 \mathrm{~h}$ of their life in the mosquito midgut. Dead parasites limit the use of standard PCR based methods to quantify live ookinete numbers during that time due to amplification of nucleic acids from both dead and viable parasites. An alternative method that addresses this limitation is quantification of mRNA that is often used as a viability marker on the basis of its rapid degradation and therefore considered to be a good indicator of live cells [29-32]. However, our data show that Plasmodium transcripts exclusively expressed in ookinetes and required for midgut invasion [33, 34] persist in mosquito midguts at least $24 \mathrm{~h}$ beyond the termination of their expression or after ookinete death. Therefore the detection and quantification of ookinetes transcripts using qrtPCR cannot exclude dead parasites, and mRNA originating from non-dead ookinetes in the midgut killed by multiple factors could lead to an overestimation of the number of ookinetes. Numerous studies have demonstrated that RNA can persist for up to a week in dead cells $[35,36]$. Due to indecisiveness of discrimination of viable cells by this technique, the use of mRNA based qrtPCR is more adapted for gene expression studies than estimating of the bacterial abundance [37].

To circumvent the indecisiveness of mRNA-based techniques, a combination of pretreatment of samples with PMA and nucleic acid detection methods have been developed as an alternative strategy [38]. This tecnique is based on DNA detection of cells with intact cell/wall membranes, the viability qPCR (v-qPCR). The PMA selectively enters dead cells and interacts with DNA preventing its amplification during PCR. Until now, the PMA-qrtPCR technique has not been tested to date in investigations involving Plasmodium viability in the mosquito vector. We demonstrate that PMA can selectively enter dead ookinetes both from in vitro cultures and in vivo, while dual staining of the mosquito midgut contents with SYBR green I (SGI) and PMA confirmed that PMA enters exclusively into dead bacterial cells. Subsequent qrtPCR of PMA treated samples effectively differentiates viable from non-viable ookinetes, and the same effect is observed on bacteria isolated from mosquito midguts.

There are several examples of successful application of the PMA-qrtPCR technique including quantification of viable oocysts of Cryptosporidium parvum [22] and trophozoites and cysts of Acanthamoeba castellani [39]. The technique has been also successfully used to quantify viable microorganisms in environmental [40-42], food [43-45] and pathological samples [46, 47]. It has been shown that the limit of detection of the PMA-qrtPCR method was at least equivalent to that obtained by using CFU values for the gram-negative bacteria Legionella pneumophila [48]. Here we show that the technique can detect as few as 10 and accurately quantify as few as 25 ookinetes.

Therefore, the PMA-qrtPCR technique can allow accurate and simultaneous monitoring of viable Plasmodium ookinetes and midgut microbiota cells during the time of mosquito midgut invasion. The ookinetes and microbiota data obtained by this method can be also used to assess the dynamics and magnitude of mosquito immune responses at a time of infection.

\section{Abbreviations}

PMA: Propidium Monoazide; PCR: Polymerase chain reaction; RT-PCR: Reverse transcriptase PCR; qrtPCR: Quantitative real-time PCR; DNA: Deoxyribonucleic acid; RNA: Ribonucleic acid; v-PCR: Viability PCR; SOAP: Secreted ookinete adhesive protein; PbSOAP: Plasmodium berghei SOAP; CTRP: Circumsporozoite and TRAP related protein; GFP: Green fluorescent protein; Ct: Threshold cycle; LoD: Limit of detection.

\section{Competing interest}

The authors declare that they have no competing interests.

\section{Authors' contribution}

Conceived and designed the experiments: TH, GKC. Performed the experiments: TH, ZG. Analyzed the data: TH, GKC. Contributed reagents/ materials/analysis tools: TH, GKC, LD. Wrote the manuscript: TH, ZG, GKC, LD All authors read and approved the final manuscript.

\section{Acknowledgments}

We thank Katarzyna Sala for technical support and Valerie Ukegbu for supply of parasite cultures. The work was supported through grants by the Wellcome Trust, WT093587MA and the BBSRC Project BB/K009338/1.

\section{Author details}

${ }^{1}$ Department of Life Sciences, Imperial College London, London, UK. ${ }^{2}$ Department of Comparative Physiology and Biometrics, University of Ghent, Ghent, Belgium. ${ }^{3}$ Costello Medical Consulting, Cambridge, UK.

Received: 10 August 2015 Accepted: 11 September 2015

Published online: 15 September 2015

\section{References}

1. Alavi Y, Arai M, Mendoza J, Tufet-Bayona M, Sinha R, Fowler K, et al. The dynamics of interactions between Plasmodium and the mosquito: a study of the infectivity of Plasmodium berghei and Plasmodium gallinaceum, and their transmission by Anopheles stephensi, Anopheles gambiae and Aedes aegypti. Int J Parasitol. 2003;33(9):933-43. Epub 2003/08/09. PubMed.

2. Ghosh A, Edwards MJ, Jacobs-Lorena M. The Journey of the Malaria Parasite in the Mosquito: Hopes for the New Century. Parasitol Today. 2000;16(5):196-201. doi:10.1016/s0169-4758(99)01626-9.

3. Vaughan JA, Hensley L, Beier JC. Sporogonic development of Plasmodium yoelii in five anopheline species. J Parasitol. 1994;80(5):674-81. Epub 1994/10/01. PubMed.

4. Vaughan JA, Noden BH, Beier JC. Population dynamics of Plasmodium falciparum sporogony in laboratory-infected Anopheles gambiae. J Parasitol. 1992;78(4):716-24. Epub 1992/08/01. PubMed.

5. Gendrin M, Christophides GK. The Anopheles Mosquito Microbiota and Their Impact on Pathogen Transmission, Anopheles mosquitoes - New insights into malaria vectors. Manguin PS, editor. http://www.intechopen. com/books/anopheles-mosquitoes-new-insights-into-malaria-vectors/theanopheles-mosquito-microbiota-and-their-impact-on-pathogentransmission. 2013.

6. Kumar S, Molina-Cruz A, Gupta L, Rodrigues J, Barillas-Mury C. A Peroxidase/ Dual Oxidase System Modulates Midgut Epithelial Immunity in Anopheles gambiae. Science. 2010;327(5973):1644-8. doi:10.1126/science.1184008. 
7. Dong Y, Das S, Cirimotich C, Souza-Neto JA, McLean KJ, Dimopoulos G. Engineered Anopheles Immunity to Plasmodium Infection. PLoS Pathog. 2011;7(12), e1002458. doi:10.1371/journal.ppat.1002458.

8. Garver LS, Bahia AC, Das S, Souza-Neto JA, Shiao J, Dong Y, et al. Anopheles Imd pathway factors and effectors in infection intensity-dependent anti-Plasmodium action. PLoS Pathog. 2012;8(6):e1002737. doi:10.1371/journal.ppat.1002737. Epub 2012/06/12. PubMed PMID: 22685401; PubMed Central PMCID: PMCPMC3369948.

9. Cirimotich CM, Dong Y, Clayton AM, Sandiford SL, Souza-Neto JA, Mulenga M, et al. Natural Microbe-Mediated Refractoriness to Plasmodium Infection in Anopheles gambiae. Science. 2011;332(6031):855-8. doi:10.1126/science.1201618.

10. Luckhart S, Vodovotz Y, Cui L, Rosenberg R. The mosquito Anopheles stephensi limits malaria parasite development with inducible synthesis of nitric oxide. Proc Natl Acad Sci U S A. 1998;95(10):5700-5.

11. Molina-Cruz A, DeJong RJ, Charles B, Gupta L, Kumar S, Jaramillo-Gutierrez $\mathrm{G}$, et al. Reactive oxygen species modulate Anopheles gambiae immunity against bacteria and Plasmodium. J Biol Chem. 2008;283(6):3217-23. doi:10.1074/jbc.M705873200. Epub 2007/12/11. PubMed.

12. Weiss B, Aksoy S. Microbiome influences on insect host vector competence. Trends Parasitol. 2011;27(11):514-22. doi:10.1016/j.pt.2011.05.001.

13. Bourgeois N, Boutet A, Bousquet PJ, Basset D, Douard-Enault C, Charachon $S$, et al. Comparison of three real-time PCR methods with blood smears and rapid diagnostic test in Plasmodium sp. infection. Clin Microbiol Infect. 2010;16(8):1305-11. doi:10.1111/j.1469-0691.2009.02933.x.

14. Farcas GA, Soeller R, Zhong K, Zahirieh A, Kain KC. Real-Time Polymerase Chain Reaction Assay for the Rapid Detection and Characterization of Chloroquine-Resistant Plasmodium falciparum Malaria in Returned Travelers. Clin Infect Dis. 2006;42(5):622-7. doi:10.1086/500134.

15. Hellyer TJ, DesJardin LE, Hehman GL, Cave MD, Eisenach KD. Quantitative analysis of mRNA as a marker for viability of Mycobacterium tuberculosis. J Clin Microbiol. 1999;37(2):290-5. Epub 1999/01/16. PubMed PMID: 9889206; PubMed Central PMCID: PMCPMC84288.

16. Hellyer TJ, DesJardin LE, Teixeira L, Perkins MD, Cave MD, Eisenach KD. Detection of viable Mycobacterium tuberculosis by reverse transcriptasestrand displacement amplification of mRNA. J Clin Microbiol. 1999;37(3):518-23. Epub 1999/02/13. PubMed PMID: 9986805; PubMed Central PMCID: PMCPMC84447.

17. Cenciarini-Borde C, Courtois S, La Scola B. Nucleic acids as viability markers for bacteria detection using molecular tools. Future Microbiol. 2009;4(1):45-64. doi:10.2217/17460913.4.1.45. Epub 2009/02/12. PubMed.

18. Nocker A, Sossa-Fernandez P, Burr MD, Camper AK. Use of propidium monoazide for live/dead distinction in microbial ecology. Appl Environ Microbiol. 2007;73(16):5111-7. doi:10.1128/aem.02987-06. Epub 2007/06/26. PubMed PMID: 17586667; PubMed Central PMCID: PMCPMC1951001.

19. Nocker A, Cheung CY, Camper AK. Comparison of propidium monoazide with ethidium monoazide for differentiation of live vs. dead bacteria by selective removal of DNA from dead cells. J Microbiol Methods. 2006;67(2):310-20. doi:10.1016/j.mimet.2006.04.015. Epub 2006/06/07. PubMed.

20. Pan Y, Breidt Jr F. Enumeration of viable Listeria monocytogenes cells by real-time $P C R$ with propidium monoazide and ethidium monoazide in the presence of dead cells. Appl Environ Microbiol. 2007;73(24):8028-31. doi:10.1128/aem.01198-07. Epub 2007/10/16. PubMed PMID: 17933922; PubMed Central PMCID: PMCPMC2168130.

21. Vesper S, McKinstry C, Hartmann C, Neace M, Yoder S, Vesper A. Quantifying fungal viability in air and water samples using quantitative $P C R$ after treatment with propidium monoazide (PMA). J Microbiol Methods. 2008;72(2):180-4. doi:10.1016/j.mimet.2007.11.017. Epub 2007/12/28. PubMed.

22. Brescia CC, Griffin SM, Ware MW, Varughese EA, Egorov Al, Villegas EN Cryptosporidium propidium monoazide-PCR, a molecular biology-based technique for genotyping of viable Cryptosporidium oocysts. Appl Environ Microbiol. 2009;75(21):6856-63. doi:10.1128/aem.00540-09. Epub 2009/09/ 15. PubMed PMID: 19749067; PubMed Central PMCID: PMCPMC2772443.

23. Coetzee M, Hunt RH, Wilkerson R, Della Torre A, Coulibaly MB, Besansky NJ. Anopheles coluzzii and Anopheles amharicus, new members of the Anopheles gambiae complex. Zootaxa. 2013;3619:246-74. Epub 2013/01/01. PubMed.

24. Franke-Fayard B, Trueman H, Ramesar J, Mendoza J, van der Keur M, van der Linden R, et al. A Plasmodium berghei reference line that constitutively expresses GFP at a high level throughout the complete life cycle. Mol Biochem Parasitol.
2004;137(1):23-33. doi:10.1016/j.molbiopara.2004.04.007. Epub 2004/07/29. PubMed.

25. Ono T, Tadakuma T, Rodriguez A. Plasmodium yoelii yoelii 17XNL constitutively expressing GFP throughout the life cycle. Exp Parasitol. 2007;115(3):310-3. doi:10.1016/j.exppara.2006.09.008. Epub 2006/10/20. PubMed PMID: 17049517; PubMed Central PMCID: PMC1779952.

26. Habtewold T, Povelones M, Blagborough AM, Christophides GK. Transmission blocking immunity in the malaria non-vector mosquito Anopheles quadriannulatus species A. PLoS Pathog. 2008;4(5):e1000070. doi:10.1371/journal.ppat.1000070. Epub 2008/05/24. PubMed PMID: 18497855; PubMed Central PMCID: PMCPMC2374904.

27. Alvarez G, Gonzalez M, Isabal S, Blanc V, Leon R. Method to quantify live and dead cells in multi-species oral biofilm by real-time PCR with propidium monoazide. AMB Express. 2013;3(1):1. doi:10.1186/2191-0855-3-1. Epub 2013/01/08. PubMed PMID: 23289803; PubMed Central PMCID: PMCPMC3549832.

28. Saadati N, Abdullah MP, Zakaria Z, Sany SB, Rezayi M, Hassonizadeh H. Limit of detection and limit of quantification development procedures for organochlorine pesticides analysis in water and sediment matrices. Chem Cent J. 2013;7(1):63. doi:10.1186/1752-153x-7-63. Epub 2013/04/09. PubMed PMID: 23561579; PubMed Central PMCID: PMCPMC3630005

29. Alifano P, Bruni C, Carlomagno M. Control of mRNA processing and decay in prokaryotes. Genetica. 1994;94(2-3):157-72. doi:10.1007/BF01443430.

30. Mendum TA, Sockett RE, Hirsch PR. The detection of Gram-negative bacterial mRNA from soil by RT-PCR. FEMS Microbiology Lett. 1998;164(2):369-73. http://femsle.oxfordjournals.org/content/164/2/369.

31. Uyttendaele $M$, van Boxstael $S$, Debevere J. PCR assay for detection of the E. coli 0157:H7 eae-gene and effect of the sample preparation method on PCR detection of heat-killed E. coli O157:H7 in ground beef. Int J Food Microbiol. 1999;52(1-2):85-95. Epub 1999/11/26. PubMed.

32. Vettraino AM, Sukno S, Vannini A, Garbelotto M. Diagnostic sensitivity and specificity of different methods used by two laboratories for the detection of Phytophthora ramorum on multiple natural hosts. Plant Pathol. 2010;59(2):289-300. doi:10.1111/j.1365-3059.2009.02209.x.

33. Dessens JT, Siden-Kiamos I, Mendoza J, Mahairaki V, Khater E, Vlachou D, et al. SOAP, a novel malaria ookinete protein involved in mosquito midgut invasion and oocyst development. Mol Microbiol. 2003;49(2):319-29. Epub 2003/06/28. PubMed.

34. Oakley MS, Kumar S, Anantharaman V, Zheng H, Mahajan B, Haynes JD, et al. Molecular factors and biochemical pathways induced by febrile temperature in intraerythrocytic Plasmodium falciparum parasites. Infect Immun. 2007;75(4):2012-25. doi:10.1128/iai.01236-06. Epub 2007/02/07. PubMed PMID: 17283083; PubMed Central PMCID: PMCPMC1865691.

35. Chimento A, Cacciola SO, Garbelotto M. Detection of mRNA by reversetranscription PCR as an indicator of viability in Phytophthora ramorum. Forest Pathol. 2012;42(1):14-21. doi:10.1111/j.1439-0329.2011.00717.x.

36. Sheridan GEC, Masters Cl, Shallcross JA, Mackey BM. Detection of mRNA by Reverse Transcription-PCR as an Indicator of Viability in Escherichia coli Cells. Appl Environ Microbiol. 1998;64(4):1313-8. PubMed.

37. Postollec F, Falentin H, Pavan S, Combrisson J, Sohier D. Recent advances in quantitative PCR (qPCR) applications in food microbiology. Food Microbiol. 2011;28(5):848-61. doi:10.1016/j.fm.2011.02.008. Epub 2011/05/17. PubMed.

38. Wagner AO, Malin C, Knapp BA, Illmer P. Removal of Free Extracellular DNA from Environmental Samples by Ethidium Monoazide and Propidium Monoazide. Appl Environ Microbiol. 2008;74(8):2537-9. doi:10.1128/aem.02288-07.

39. Fittipaldi M, Pino Rodriguez NJ, Adrados B, Agustí G, PeÑUela G, MoratÓ J, et al. Discrimination of Viable Acanthamoeba castellani Trophozoites and Cysts by Propidium Monoazide Real-Time Polymerase Chain Reaction. J Eukaryot Microbiol. 2011;58(4):359-64. doi:10.1111/j.1550-7408.2011.00557.x.

40. Nocker A, Richter-Heitmann T, Montijn R, Schuren F, Kort R. Discrimination between live and dead cellsin bacterial communities from environmental water samples analyzed by 454 pyrosequencing. Int Microbiol. 2010;13(2):59-65. Epub 2010/10/05. PubMed.

41. Wagner AO, Malin C, Knapp BA, Illmer P. Removal of free extracellular DNA from environmental samples by ethidium monoazide and propidium monoazide. Appl Environ Microbiol. 2008;74(8):2537-9. doi:10.1128/aem.02288-07. Epub 2008/02/26. PubMed PMID: 18296534; PubMed Central PMCID: PMCPMC2293149.

42. Taskin B, Gozen AG, Duran M. Selective quantification of viable Escherichia coli bacteria in biosolids by quantitative PCR with propidium monoazide modification. Appl Environ Microbiol. 2011;77(13):4329-35. 
doi:10.1128/aem.02895-10. Epub 2011/05/24. PubMed PMID: 21602375; PubMed Central PMCID: PMCPMC3127682.

43. Li B, Chen JQ. Development of a sensitive and specific qPCR assay in conjunction with propidium monoazide for enhanced detection of live Salmonella spp. in food. BMC Microbiol. 2013;13:273. doi:10.1186/1471-2180-13-273. Epub 2013/12/03. PubMed.

44. Liang N, Dong J, Luo L, Li Y. Detection of viable Salmonella in lettuce by propidium monoazide real-time PCR. J Food Sci. 2011;76(4):M234-7. doi:10.1111/j.1750-3841.2011.02123.x. Epub 2012/03/16. PubMed.

45. Banihashemi A, Van Dyke MI, Huck PM. Long-amplicon propidium monoazidePCR enumeration assay to detect viable Campylobacter and Salmonella. J Appl Microbiol. 2012;113(4):863-73. doi:10.1111/j.1365-2672.2012.05382.x. Epub 2012/07/04. PubMed.

46. Yasunaga A, Yoshida A, Morikawa K, Maki K, Nakamura S, Soh I, et al. Monitoring the prevalence of viable and dead cariogenic bacteria in oral specimens and in vitro biofilms by qPCR combined with propidium monoazide. BMC Microbiol. 2013;13:157. doi:10.1186/1471-2180-13-157. Epub 2013/07/16. PubMed PMID: 23848601; PubMed Central PMCID: PMCPMC3717283.

47. Rogers GB, Cuthbertson L, Hoffman LR, Wing PA, Pope C, Hooftman DA, et al. Reducing bias in bacterial community analysis of lower respiratory infections. ISME J. 2013;7(4):697-706. doi:10.1038/ismej.2012.145. Epub 2012/ 11/30. PubMed PMID: 23190732; PubMed Central PMCID: PMCPMC3603400.

48. Chang B, Taguri T, Sugiyama K, Amemura-Maekawa J, Kura F, Watanabe H. Comparison of ethidium monoazide and propidium monoazide for the selective detection of viable Legionella cells. Jpn J Infect Dis. 2010;63(2):119-23. Epub 2010/03/25. PubMed.

\section{Submit your next manuscript to BioMed Central and take full advantage of:}

- Convenient online submission

- Thorough peer review

- No space constraints or color figure charges

- Immediate publication on acceptance

- Inclusion in PubMed, CAS, Scopus and Google Scholar

- Research which is freely available for redistribution 